\title{
Final results of the US humanitarian device exemption study of the low-profile visualized intraluminal support (LVIS) device
}

\author{
David Fiorella, ${ }^{1}$ Adam Arthur, ${ }^{2}$ Alan Boulos, ${ }^{3}$ Orlando Diaz, ${ }^{4}$ Pascal Jabbour, ${ }^{5}$ \\ Lee Pride, ${ }^{6}$ Aquilla S Turk, ${ }^{7}$ Henry H Woo, ${ }^{1}$ Colin Derdeyn, ${ }^{8}$ John Millar, ${ }^{9}$ \\ Andrew Clifton ${ }^{10}$
}

${ }^{1}$ Department of Neurosurgery, State University of New York at Stony Brook, Stony Brook, New York, USA

${ }^{2}$ Methodist Hospital, Memphis, Tennessee, USA

${ }^{3}$ Albany Medical Center, Albany, New York, USA

${ }^{4}$ Methodist Houston, Houston, Texas, USA

${ }^{5}$ Department of Neurological Surgery, Thomas Jefferson Hospital, Philadelphia, Pennsylvania, USA

${ }^{6}$ Department of Neuroradiology, University of Texas Southwestern, Dallas, Texas, USA

7 Medical University of South Carolina, Charleston, South Carolina, USA

${ }^{8}$ Washington University, St. Louis, Missouri, USA

${ }^{9}$ Department of Neuroradiology, Wessex Neurological Centre, Southampton, UK

${ }^{10}$ St Georges Hospital Medical School, London, UK

\section{Correspondence to} Dr David Fiorella, Department of Neurological Surgery, Stony Brook University Medical

Center, Cerebrovascular Center, Health Sciences Center T-12 080, Stony Brook NY 11794-8122, USA; david.fiorella@sbumed.org

Received 30 June 2015 Revised 25 August 2015 Accepted 31 August 2015 Published Online First 21 September 2015

CrossMark

To cite: Fiorella $D$,

Arthur A, Boulos A, et al.

I Neurolntervent Surg

2016:8:894-897.

\section{ABSTRACT}

Introduction The low-profile visualized intraluminal support (LVIS) device is a new, braided, intracranial microstent designed for stent-assisted coiling.

Objective To present the results of a single-arm, prospective, multicenter trial of the LVIS for treatment of wide-necked intracranial aneurysms.

Methods 31 patients with unruptured, wide-necked (neck $\geq 4 \mathrm{~mm}$ or dome:neck ratio $\leq 2$ ) intracranial aneurysms were treated with the LVIS device and bare platinum coils at six US centers (investigational device exemption G110014). Clinical follow-up was conducted at 30 days and 6 months. Angiographic follow-up was performed at 6 months. The primary safety endpoint was any major stroke or death within 30 days or major ipsilateral stroke or neurological death within 6 months. 'Probable benefit' was defined as $\geq 90 \%$ angiographic occlusion at 6 months. An independent core laboratory adjudicated the angiographic results. An independent clinical events committee adjudicated the clinical endpoints.

Results Average aneurysm size was $7.2 \mathrm{~mm}$ (SD 3.8) and average neck width was $4.6 \mathrm{~mm}$ (SD 1.8). $68 \%$ of patients had a dome:neck ratio $\leq 2$. LVIS placement was technically successful in 29/31 patients (93.5\%). No primary safety endpoints occurred during the study $(0 \%)$. No patient had a higher modified Rankin Score at 6 months than at baseline. 26/28 (92.9\%) treated aneurysms with 6-month angiographic follow-up demonstrated $\geq 90 \%$ angiographic occlusion. 21/28 (75\%) were completely occluded at follow-up. Conclusions The LVIS device facilitated the coil embolization of wide-necked intracranial aneurysms with high rates of technical success, an excellent safety profile, and very high rates of complete and near-complete occlusion at follow-up.

Trial registration number NCT01541254.

\section{INTRODUCTION}

The low-profile visualized intraluminal support (LVIS) device is a flexible, braided microstent designed for the stent-assisted coiling of wide-necked intracranial aneurysms. We present the results of a multicenter prospective, single-arm trial of the LVIS device.

\section{METHODS}

\section{Enrollment}

A total of 36 subjects were enrolled at six participating USA centers from March to November
2012, with 31 subjects treated (or treatment was attempted) with the LVIS device at multiple sites under an investigational device exemption (IDE G110014, conditionally approved on May 4, 2011). The IDE study protocol was approved by the institutional review boards at all participating institutions. The 6-month follow-up for the last subject was completed in June 2013.

\section{Study parameters}

Subjects $18-80$ years of age with wide-necked saccular aneurysms ranging in size between 4 and $20 \mathrm{~mm}$ and arising from a parent artery with a diameter ranging between 2.5 and $4.5 \mathrm{~mm}$, were included in the study. Wide neck was defined as a an absolute neck width $\geq 4 \mathrm{~mm}$ or a dome:neck ratio of $\leq 2$.

Subjects with ruptured aneurysms, intracranial masses, abnormal coagulation, contraindications to contrast administration or antiplatelet drugs, nickel-titanium allergy, cardiac disorders resulting in an increased risk of stroke, or who were unable to complete follow-up were excluded.

The primary endpoints for the study were:

1. Any major stroke or death within 30 days or major ipsilateral stroke or neurological death within 6 months (safety endpoint).

2. Successful aneurysm treatment with the LVIS device as defined by angiographic occlusion of $\geq 90 \%$ at 6 months as determined by an independent core laboratory (probably benefit endpoint).

The secondary endpoints were:

1. Device- and procedure-related serious adverse events.

2. Successful delivery of the LVIS device.

3. Parent artery patency at 6 months.

4. Stent migration at 6 months.

5. Significant $(>50 \%)$ stenosis of the treated artery at 6 months.

6. Unplanned embolization coiling within 6 months.

Clinical follow-up was performed at discharge, 30 days ( \pm 7 days) and 6 months ( \pm 4 weeks). Angiographic follow-up was performed at 6 months. Subjects who were enrolled but not treated because of a failed device deployment were monitored for 1 week. Enrollment was declared at the time the patient signed the informed consent document for the study. 


\section{LVIS device description}

The LVIS device is a self-expanding nickel-titanium, single-wire braid, compliant, closed-cell, retrievable, intracranial microstent designed for the stent-assisted coil embolization of wide-necked intracranial aneurysms. The device has two forms, the LVIS (figure $1 \mathrm{~A}$ ), which is compatible with a $0.021^{\prime \prime}$ internal diameter (ID) Headway microcatheter (Microvention/Terumo, Tustin, California, USA) and is recommended for vessels sized 3.0$4.5 \mathrm{~mm}$, and the LVIS Junior (figure 1B), which is compatible with a standard $0.017^{\prime \prime}$ ID microcatheter or dual lumen balloon microcatheter (Scepter C or XC, Microvention/Terumo) and is recommended for vessels sized $2.5-3.0 \mathrm{~mm}$. The LVIS has an unconstrained outer diameter ranging between 3.5 and $5.5 \mathrm{~mm}$ and comes in unconstrained lengths ranging between 15 and $25 \mathrm{~mm}$. The LVIS Junior has an unconstrained outer diameter of $3.5 \mathrm{~mm}$ and comes in unconstrained lengths ranging between 15 and $32 \mathrm{~mm}$. Owing to the braided design, the in situ working length is a function of the degree of foreshortening that occurs within the parent artery, which is dependent upon the device size selected relative to the vessel diameter.

LVIS is provided as a stent, constrained within an introducer sheath, mounted upon a delivery wire. The device is loaded into the microcatheter and delivered to the desired site of deployment. Device deployment occurs as the expanded device is released from the delivery wire. The stent is fully re-constrainable after deployment of up to $80 \%$ of its length.

\section{LVIS procedure}

Patients were pretreated with aspirin (81-325 mg/day by mouth given 3-7 days before the procedure) and clopidogrel $(75 \mathrm{mg} /$ day by mouth given at least 5 days before the procedure or as a loading dose of $600 \mathrm{mg}$ given at least $2 \mathrm{~h}$ before the procedure).
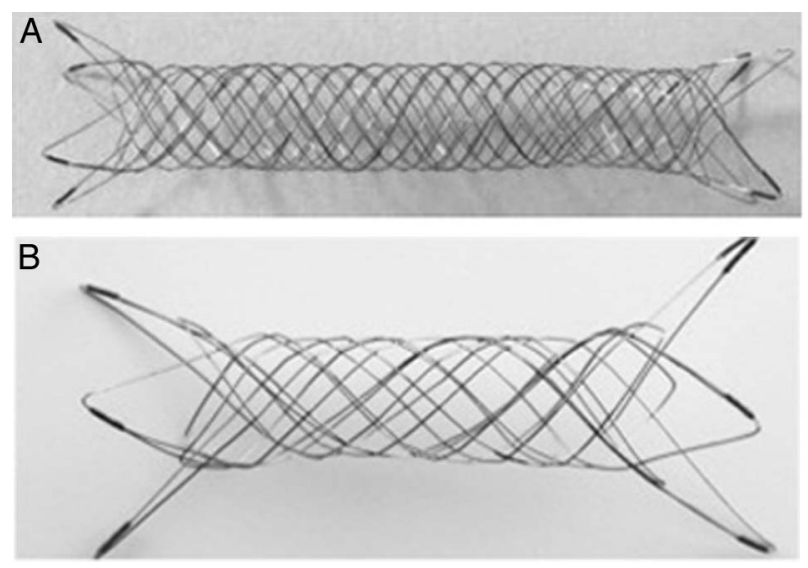

Figure 1 The low-profile visualized intraluminal support (LVIS) device (A) is braided from 16 passes with a single wire. The ends have four flares. Proximally, each of the four flares has a radiopaque marker. Distally two of the flares have two radiopaque markers. There are also two radiopaque strands running along the length of the device. The LVIS is indicated for vessels ranging between 3.0 and $4.5 \mathrm{~mm}$ and is available with fully expanded diameters of $3.5,4.5$, and $5.5 \mathrm{~mm}$. The cell size between braids is $1.0 \mathrm{~mm}$; however, the braids are mobile and can splay to accommodate a microcatheter. The LVIS is delivered through a Headway 021 microcatheter. The LVIS Junior device (B) is braided from 12 passes with a single wire. The ends have three flares, each with a radiopaque marker. There are also three radiopaque strands running along the length of the device. The LVIS Junior is indicated for vessels ranging between 2.5 and $3.0 \mathrm{~mm}$ and has a fully expanded diameter of $3.5 \mathrm{~mm}$. The cell size between braids is $1.5 \mathrm{~mm}$. The LVIS Junior is delivered through a Headway 017 microcatheter.
There was no mandate for preprocedural platelet function testing within the protocol and these data were not systematically collected. During the procedure patients were administered IV heparin to maintain an activated clotting time close to 250 $300 \mathrm{~s}$ throughout. The clotting time was monitored at $60 \mathrm{~min}$ intervals during the procedure. After the procedure, patients were maintained on 81-325 mg aspirin/day and $75 \mathrm{mg}$ of clopidogrel/day for at least 3 months with one antiplatelet agent (typically aspirin) recommended for life.

Most procedures were performed through a 6 French femoral access using standard commercially available guiding catheters and sheaths. The LVIS device could be used in several ways within the context of the study:

1. Standard trans-stent coiling: LVIS deployed across the aneurysm neck through a standard 017 (LVIS Junior) or 021 (LVIS) Headway microcatheter. The deployed LVIS stent is then traversed with a microcatheter to allow for aneurysm coiling.

2. Microcatheter jailing: after the introduction of a microcatheter into the aneurysm, the LVIS device is deployed (through a second Headway microcatheter) across the aneurysm neck. The aneurysm is then coiled through the primary microcatheter.

3. Stent placement after coiling: after coil embolization of the aneurysm (standard coiling or balloon-assisted coiling), the LVIS device is deployed across the aneurysm neck.

4. Stent deployment through a dual lumen hypercompliant balloon catheter: the aneurysm is catheterized with a standard microcatheter and a balloon microcatheter is positioned across the aneurysm neck. After balloon-assisted coiling, the LVIS junior device is deployed through the lumen of the balloon microcatheter to secure the coil mass.

All aneurysms were embolized with standard bare platinum coils.

Angiography in standard branch projections and the working angles for coil embolization were performed before and after coil embolization and at the 6-month follow-up examination. All angiographic data were provided as DICOM (Digital Imaging and Communications in Medicine) files for review by an independent core laboratory. Aneurysms were characterized as showing either complete occlusion (100\% embolization), near-complete occlusion (90-99\%), or partial occlusion $(<90 \%)$. Parent artery stenosis was defined as $>50 \%$ luminal loss at follow-up by the method defined in the WASID study (Warfarin-Aspirin Symptomatic Intracranial Disease Study).

An independent clinical events committee/data safety monitoring committee that met regularly during the study performed data monitoring and event adjudication.

\section{RESULTS}

\section{Subjects and aneurysm characteristics}

A total of 36 patients were enrolled, of whom 31 were treated during the study (23 women, average age 58.6 years). Five patients were excluded before treatment for the following reasons: withdrew consent, nickel-titanium allergy discovered, gastrointestinal bleed before procedure, enrolled after study closed, coiling of the aneurysm achieved without the need for a stent. Of the 31 patients enrolled and undergoing treatment, 30 had the study device implanted, 31 were seen at the 30 -day follow-up, 29 were seen at the 6-month clinical follow-up, and 28 underwent angiographic follow-up at 6 months. Average aneurysm size was $7.2 \mathrm{~mm}$ (SD 3.8) and average neck width was $4.6 \mathrm{~mm}$ (SD 1.8). Sixty-eight per cent of patients had a dome: neck ratio $\leq 2$. Of the treated aneurysms, five were located 
within the posterior circulation (basilar apex, basilar trunk, superior cerebellar artery, and posterior inferior cerebellar artery). The remaining aneurysms arose from the anterior circulation (internal carotid artery $(\mathrm{n}=20)$, middle cerebral artery $(\mathrm{n}=4)$, anterior cerebral artery $(\mathrm{n}=1)$ ) (table 1$)$. Four LVIS Junior devices were used, and the remaining patients underwent treatment with LVIS.

\section{Device deployment and delivery}

LVIS delivery and deployment was technically successful in $29 / 31$ patients $(93.5 \%)$. In one patient the device could not be deployed within an internal carotid artery and treatment was ultimately accomplished with a different microstent. In this case, the investigator could not achieve an optimal position of the stent across the aneurysm neck. For this reason, the LVIS was reconstrained and removed. In the other patient, the proximal aspect of the LVIS did not open fully after deployment. Despite this, a microcatheter was easily manipulated across the stent, and a technically successful treatment of the aneurysm was still achieved. In 11 patients, trans-stent coiling was performed (figure 2). In 10 patients a jailing technique was used. In eight patients the aneurysm was coiled (with or without balloon assistance) followed by stenting. In one patient, balloon-assisted coiling with a Scepter XC was followed by deployment of the stent through the balloon catheter. In one case both trans-stent and jailing techniques were used. In one case, an alternative stent was used because the LVIS could not be adequately deployed across the aneurysm neck within the parent artery.

\section{Primary endpoints}

No primary safety endpoints occurred during the study $(0 \%)$. No patient had a higher modified Rankin Score at 6 months than at baseline.

Twenty-six of $28(92.9 \%)$ treated aneurysms with 6-month angiographic follow-up demonstrated $\geq 90 \%$ angiographic occlusion. Twenty-one of the 28 (75\%) were completely occluded at follow-up.

\section{Secondary endpoints}

Two probable device/procedure-related serious neurological adverse events occurred during the study. One patient experienced lightheadedness on postprocedure day 3. MRI showed several tiny punctate foci of restricted diffusion, which were felt to be incidental. This event was adjudicated as minor stroke with complete resolution of symptoms at clinical follow-up. A second patient experienced transient worsening of a baseline gait imbalance with headache 4 months after the procedure.

Table 1 Aneurysm locations

\begin{tabular}{llc}
\hline Location & N & Percentage \\
\hline Anterior communicating & 1 & 3 \\
Basilar tip & 1 & 3 \\
Basilar trunk & 1 & 3 \\
Carotid ophthalmic & 6 & 19 \\
Carotid cavernous & 2 & 6 \\
Middle cerebral & 4 & 13 \\
Posterior communicating & 5 & 16 \\
Posterior inferior cerebellar & 2 & 6 \\
Superior cerebellar & 1 & 3 \\
Superior hypophyseal & 8 & 26 \\
\hline
\end{tabular}

Plavix had been discontinued 1 week previously. MR with diffusion demonstrated two tiny punctate foci of diffusion restriction in the distribution of the treated vessel. Angiography demonstrated complete patency of the LVIS device and occlusion of the aneurysm. Plavix was resumed and all symptoms resolved. This event was also adjudicated as a delayed minor stroke with complete resolution of symptoms at clinical follow-up.

No cases of in-stent stenosis or stent occlusion were seen at angiographic follow-up. No instances of stent migration were reported.

\section{DISCUSSION}

The most important finding of this study is that the treatment of wide-necked cerebral aneurysms is both feasible and safe with the LVIS device.

The advent of flexible, microcatheter-delivered, intracranial microstents has increased the scope of cerebral aneurysms amenable to endovascular treatment. ${ }^{1-3}$ The first generation of intracranial, coil-assist microstents (Neuroform, Stryker Therapeutics, Minneapolis, Minnesota, USA) had an opencelled design and were delivered initially through a coaxial, $0.027^{\prime \prime}$ ID microcatheter system. Because of the open-cell design, these stents were not re-constrainable once deployment was initiated. The second generation of microstents were closed cell (Enterprise, Codman Neurovascular, Raynham, Massachusetts, USA; Solitaire ev3/Covidien, Irvine, California, USA), allowing re-constrainment and repositioning after partial (Enterprise) or full (Solitaire) deployment. ${ }^{4-6}$ In addition, these closed-cell stents were deliverable through lower-profile $0.021^{\prime \prime}$ ID microcatheters. Unlike the predecessor devices, which were produced by a laser cutting a stent pattern from a Nitinol hypotube, the third generation of coil-assist microstents (LVIS and Junior, Microvention/Terumo, Tustin, California, USA; Leo, Leo +, and Baby Leo, BALT Extrusion, Montmorency, France) are produced by braiding individual strands of Nitinol onto a mandrel. ${ }^{7-9}$ This design allowed some of these stents (LVIS Junior and Baby Leo) to be delivered through even lower-profile microcatheters, requiring an ID of only $0.0165^{\prime \prime}$.

The braided morphology potentially allows greater conformability, apposition to the vessel wall, and stability. The tines of the open-celled Neuroform stent typically project into the vessel lumen in curved vascular segments. In addition, when the aneurysm arises from the outer curvature of the vessel, the cells can splay open and potentially allow coil herniation into the parent artery. ${ }^{10}{ }^{11}$ The closed-cell Enterprise stent has been shown to ovalize in curved vascular segments. ${ }^{11}$ In addition, when deployed in vascular segments with a significant diameter discrepancy (eg, basilar artery to posterior cerebral artery), stent migration can occur. ${ }^{12}{ }^{13}$ When deployed properly, the braided stents open to oppose the vessel wall closely on both the inner and outer curvature of the vessel. When optimal apposition is not achieved with the original deployment, it can typically be corrected with gentle expansion of the device with a hypercompliant balloon. As the braided configuration can either foreshorten or elongate depending on the diameter of the parent artery in which it is deployed, there are no disproportionate radial forces exerted upon the device which could cause migration after deployment in vascular segments of varying diameter.

In this study, application of the LVIS device was safe with no major neurological morbidity and no mortality reported. The LVIS devices also demonstrated acceptable efficacy with $>90 \%$ of aneurysms showing near complete occlusion (95-99\%) and $75 \%$ showing complete $(100 \%)$ occlusion. The results achieved with the LVIS device are comparable to those reported in the 

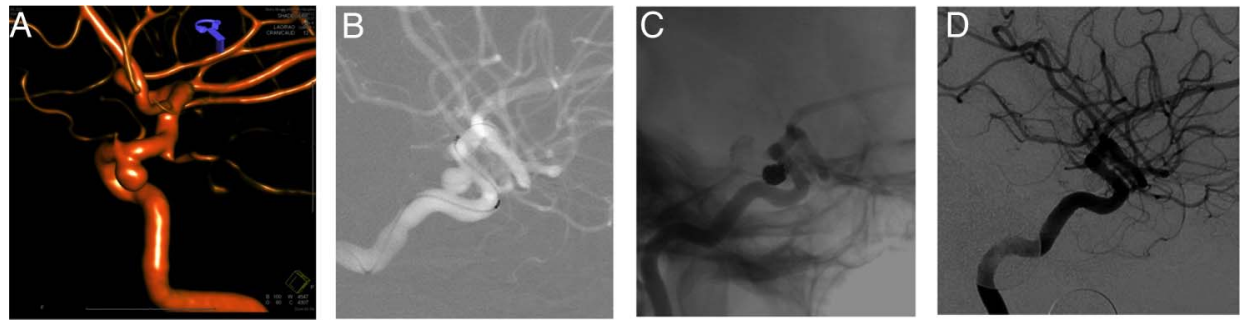

Figure 2 Rotational angiogram (A) demonstrates a wide-necked aneurysm arising from the posterior wall of the internal carotid artery. Angiographic roadmap (B) shows the low-profile visualized intraluminal support (LVIS) device constrained within the Headway 021 microcatheter and positioned across the aneurysm neck. Native angiographic image (C) demonstrates a dense coil mass within the aneurysm and the LVIS device in optimal position across the neck of the aneurysm. A subtracted angiographic image 6 months later (D) demonstrates complete occlusion of the aneurysm and no stenosis within the construct.

initial studies of the Neuroform (69\% complete occlusion, 31\% near complete occlusion) and Enterprise stents (36\% complete occlusion, 28\% near complete, and 36\% partial occlusion). The progression to complete occlusion with the LVIS device was particularly impressive, with only $17 \%$ of aneurysms showing complete occlusion immediately after treatment, increasing to $75 \%$ at the 6-month follow-up.

The LVIS Junior offers particular advantages and versatility given its deliverability though an $0.0165^{\prime \prime}$ ID microcatheter. First, it is possible to perform a jailing technique through standard $6 \mathrm{~F}$ guiding catheters. Second, it is possible to perform a balloon-assisted coiling with a standard dual lumen hypercompliant balloon and then, if necessary, place an LVIS Junior through the wire lumen of the balloon catheter. This avoids the microwire exchanges typically required for the exchange of a balloon catheter for a standard 0.021 or $0.027^{\prime \prime}$ ID stent delivery microcatheter. Finally, although not performed in this study, the LVIS Junior provides a means of creating the second limb of a Y-construct while only crossing the initially placed stent with an 017 microcatheter. Other microstents are delivered through larger microcatheters (021 or 027 ), which have a greater risk of displacing or distorting the primary limb during traversal.

In summary, the braided LVIS devices represent the third generation of coil-assist stents. This prospective, multicenter, singlearm study demonstrates the feasibility and safety of LVIS for the treatment of intracranial aneurysms.

Contributors All authors made substantial contributions to the conception and design, acquisition of data, or analysis and interpretation of data.

Funding This research was funded by Microvention/Terumo Inc. SUNY at Stony Brook receives a grant to support the LVIS HDE study.

Competing interests DF received institutional salary support for his role as National Study principal investigator. AST and AA received funds for research and or consulting funds from Microvention, Inc.
Ethics approval All local institutional review boards approved the study. Provenance and peer review Not commissioned; externally peer reviewed.

\section{REFERENCES}

1 Fiorella D, Albuquerque FC, Deshmukh VR, et al. Usefulness of the neuroform stent for the treatment of cerebral aneurysms: results at initial (3-6-mo) follow-up. Neurosurgery 2005;56:1191-201; discussion 1201-1192.

2 Fiorella D, Albuquerque FC, Han P, et al. Preliminary experience using the neuroform stent for the treatment of cerebral aneurysms. Neurosurgery 2004;54:6-16; discussion 16-17.

3 Fiorella D, Albuquerque FC, Woo $\mathrm{H}$, et al. Neuroform stent assisted aneurysm treatment: evolving treatment strategies, complications and results of long term follow-up. J Neurointerv Surg 2010;2:16-22.

4 Mocco J, Snyder KV, Albuquerque FC, et al. Treatment of intracranial aneurysms with the enterprise stent: a multicenter registry. J Neurosurg 2009;110:35-9.

5 Klisch J, Clajus C, Sychra V, et al. Coil embolization of anterior circulation aneurysms supported by the solitaire $A B$ neurovascular remodeling device. Neuroradiology 2010;52:349-59.

6 Klisch J, Eger C, Sychra V, et al. Stent-assisted coil embolization of posterior circulation aneurysms using Solitaire AB: preliminary experience. Neurosurgery 2009;65:258-66; discussion 266.

7 Spiotta AM, Miranpuri A, Chaudry Ml, et al. Combined balloon stent technique with the Scepter $C$ balloon and low-profile visualized intraluminal stent for the treatment of intracranial aneurysms. J Neurointerv Surg 2013;5(Suppl 3):iii79-82.

8 Turner RD, Turk A, Chaudry I. Low-profile visible intraluminal support device: immediate outcome of the first three US cases. J Neurointerv Surg 2013;5:157-60.

9 Pardo MI, Pumar JM, Blanco $M$, et al. Medium-term results using the leo self-expanding stent in the treatment of complex intracranial aneurysms. Neuroradiol J 2008;21:704-11.

10 Benndorf G, Claus B, Strother CM, et al. Increased cell opening and prolapse of struts of a neuroform stent in curved vasculature: value of angiographic computed tomography: technical case report. Neurosurgery 2006;58(4 Suppl 2):ONS-E380; discussion ONS-E380.

11 Ebrahimi N, Claus B, Lee CY, et al. Stent conformity in curved vascular models with simulated aneurysm necks using flat-panel CT: an in vitro study. AJNR Am J Neuroradiol 2007;28:823-9.

12 Dashti SR, Fiorella D, Toledo MM, et al. Proximal migration and compaction of an enterprise stent into a coiled basilar apex aneurysm: a posterior circulation phenomenon? J Neurointerv Surg 2010;2:356-8.

13 Kelly ME, Turner RDt, Moskowitz SI, et al. Delayed migration of a self-expanding intracranial microstent. AJNR Am J Neuroradio/ 2008;29:1959-60. 